\title{
An Approach to a Chiral Cycloalkanone-Mediated Asymmetric Epoxidation of Stilbene with Oxone ${ }^{\circledR}$
}

\author{
Koichiro Matsumoto and Kiyoshi TоміокA* \\ Graduate School of Pharmaceutical Sciences, Kyoto University, Sakyo-ku, Kyoto 606-8501, Japan. \\ Received July 30, 2001; accepted September 27, 2001
}

\begin{abstract}
Chiral and C2-symmetric seven-membered cycloalkanones $2-6$ bearing 1,2-diphenylethane-1,2-diamine and cyclohexane-1,2-diamine backbones were synthesized and evaluated their asymmetry inductive behaviours in an asymmetric epoxidation of stilbene with oxone ${ }^{\circledR}$. Although the reaction of the ketones 2 and 3 of a 1,2diphenylethane-1,2-diamine backbone gave stilbene oxide in trace to $31 \%$ yield, those of the ketones $4-6$ of a cyclohexane-1,2-diamine backbone gave the epoxide in satisfactorily high yield up to $98 \%$. It is noteworthy that both reactions with use of stoichiometric and substoichiometric amounts of a ketone 4 gave the epoxide in the essentially same enantioselectivity, 17 and 18\%. Eleven-membered cyclic ketones 7 and 8 bearing a binaphthalene backbone were also synthesized and examined their behaviours, while the enantioselectivity turned out to be marginal.
\end{abstract}

Key words epoxidation; olefin; oxone ${ }^{\circledR}$; ketone; asymmetric synthesis; stilbene

In these years we have been involved in the development of external chiral ligands effective for a variety type of catalytic asymmetric reactions. ${ }^{1,2)}$ As an approach toward asymmetric epoxidation of an olefin with oxone ${ }^{\circledR},{ }^{3)}$ we designed a chiral ketone 1 for a precursor to a dioxilane (Fig. 1). ${ }^{4)}$ The chiral ketone 1 is characteristic by a 1,2-diphenylethane-1,2diamine backbone ${ }^{5)}$ and benzenesulfonamide moiety as an electron-withdrawing group for electrophilic activation of a ketone carbonyl group as well as prevention of BaeyerVilliger oxidative rearrangement of a dioxilane. ${ }^{6}$ However, enantioselectivity was relatively low, $30 \%$ ee for epoxidation of stilbene, even though the sense of enantiofacial differentiation was exactly the same as we predicted. ${ }^{7)}$ The corresponding dioxilane A, generated from $\mathbf{1}$ and oxone ${ }^{\circledR}$, is anticipated to take a rigid conformation $\mathrm{B}$ due to steric reason, of which chiral environment is expected to be good for enantiofacial selection (Fig. 2). Since modification of the benzene ring of arylsulfonamide is one possibility to tune up the structure 1, new chiral ketones $\mathbf{2}$ and $\mathbf{3}$ bearing bulky 2,4,6trimethyl and 2,4,6-triisopropylbenzenesulfonamides were synthesized and examined their efficiency. Three cyclohexyl versions $4-6$ were also prepared and evaluated in an epoxidation of stilbene. Other than seven-membered cycloalkanones, the 11-memberd cycloalkanones 7 and 8 bearing a binaphthyl backbone were prepared and also examined their attitudes toward an asymmetric epoxidation of stilbene. It is noteworthy that epoxidation of stilbene with $\mathbf{4}$ gave stilbene oxide in reasonably high yield and almost the same enantioselectivity, regardless of whether stoichiometric (1.0 eq) or substoichiometric $(0.3 \mathrm{eq})$ amount of $\mathbf{4}$ was used.

Synthesis of the 7-Membered Chiral and C2 Symmetric Cycloalkanones 2-6 The ketones $\mathbf{2}$ and $\mathbf{3}$ were readily prepared in three steps starting from commercially available $(S, S)$-diamine 9 (Chart 1). According to the reported procedure, 9 was arylsulfonated with the corresponding chlorides in methylene chloride giving 10a, $\mathbf{b}{ }^{8)}$ Cyclization of 10 was carried out with 3-iodo-2-iodomethylpropene in $N, N$-dimethylformamide (DMF) to afford olefins 11a, b, which were then ozonolyzed to the ketones $\mathbf{2}$ and $\mathbf{3}$ (Chart 1).

The chiral ketones 4-6 bearing a cyclohexanediamine backbone were prepared by the same reaction sequence from
$(R, R)$-diamine $\mathbf{1 2}^{9)}$ (Chart 2). The phosphonamide 6 was designed expecting more bulkiness than $\mathbf{4}$ and $\mathbf{5}$ around the nitrogen.

Synthesis of the 11-Membered Chiral Cycloalkanones 7 and 8 The ketone 7 was readily prepared from a binaph-

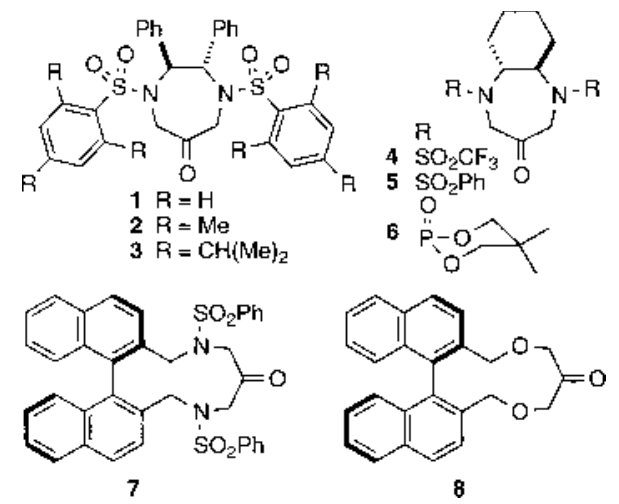

Fig. 1. Chiral Ketones for Asymmetric Epoxidation

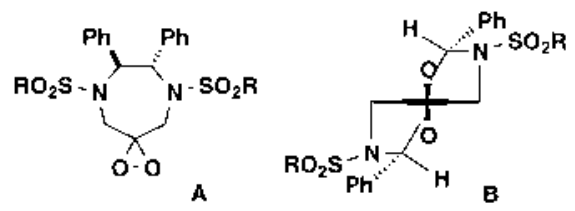

Fig. 2. Anticipated Structures of Dioxilane A and B of $\mathbf{1}-\mathbf{3}$
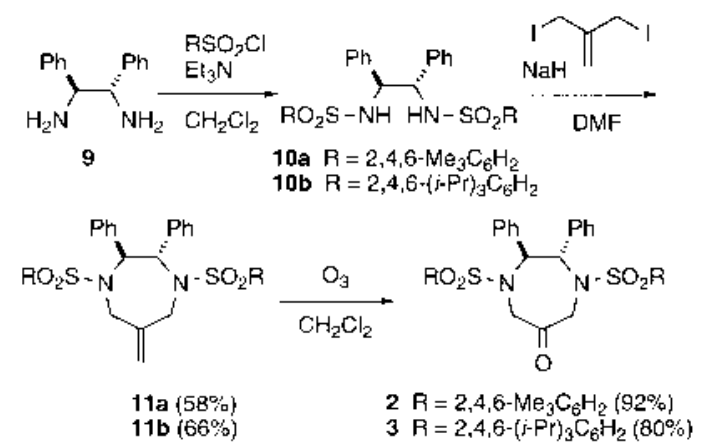

Chart 1. Synthesis of $\mathbf{2}$ and $\mathbf{3}$ from a Diamine $\mathbf{9}$ 
thalenedicarboxylic acid $\mathbf{1 5}^{\mathbf{1 0})}$ under the standard conditions (Chart 3). The diacid was converted to a diamine 16b in $69 \%$ yield, which was then subjected to the standard three-step procedure for the preparation of cycloalkanone. The target ketone 7 was obtained in satisfactorily high yield.

On the other hand, the reaction of a diol $\mathbf{1 9}^{11)}$ with diiodide did not proceed to cyclize. However, reaction with dichloride gave mono-alkylation product $\mathbf{2 0}$ in $92 \%$ yield (Chart 4 ). Treatment of $\mathbf{2 0}$ with sodium hydride in DMF gave the desired cyclized-olefin 21 in $15 \%$ yield, which was then con-

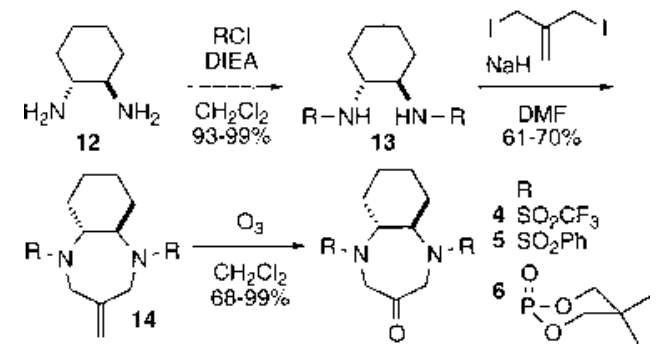

Chart 2. Three Step Synthesis of the Chiral Ketones 4-6

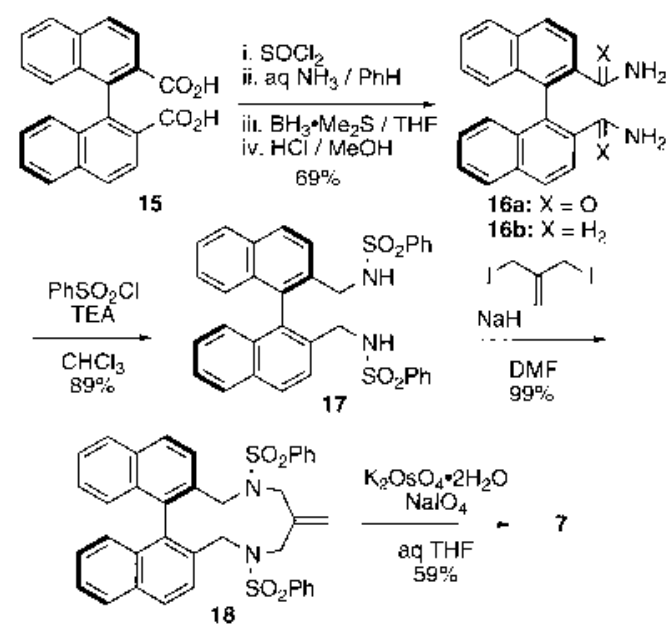

Chart 3. Synthesis of Chiral Ketone 7 from a Carboxylic Acid $\mathbf{1 5}$ verted to an alkanone $\mathbf{8}$ in $99 \%$ yield.

Asymmetric Epoxidation of Stilbene with Oxone ${ }^{\circledR}$ Using Chiral Cycloalkanones 2-8 The cycloalkanones 2 and $\mathbf{3}$ were evaluated as the precursors of the dioxilane $\mathrm{A}$ in the asymmetric epoxidation reaction of trans-stilbene. According to the previously reported reaction of $1,{ }^{7)}$ we first examined the reaction using one equivalent of 2 in 1,4-dioxane and acetonitrile as a solvent (Table 1, entries 1-3). The chemical yield of stilbene oxide was not improved and more surprisingly the enantioselectivity was quite marginal. The ketone $\mathbf{3}$ bearing more bulky triisopropylbenzenesulfonyl group did not mediated the epoxidation and gave only a trace amount of the epoxide (entry 4). On the other hand, the ketones 4-6 bearing a cyclohexyl backbone behaved more favourably (entries 5-10). Trifluoromethanesulfonylamide 4 promoted the reaction and gave the epoxide in 49 and $98 \%$ yields in dioxane and acetonitrile, respectively (entries 5, 6). The enantioselectivities were 14 and $17 \%$. More interestingly, substoichiometric $(0.3 \mathrm{eq})$ amount of $\mathbf{4}$ gave stilbene oxide in $78 \%$ yield and $18 \%$ ee (entry 7 ). Benezenesulfonamide 5 gave the product in 53\% chemical yield and 7\% ee (entry 8). Phosphonylamide 6 expecting more bulkiness gave the product in $71 \%$ chemical yield and $20 \%$ ee.

The ketones 7 and 8 bearing a binaphthyl and 11-membered backbone mediated the epoxidation reaction and gave the epoxide in relatively high chemical yields (entries 11 , 12). However, the enantioselectivity was far away from our satisfaction. It is also very disappointing to find that Baeyer-

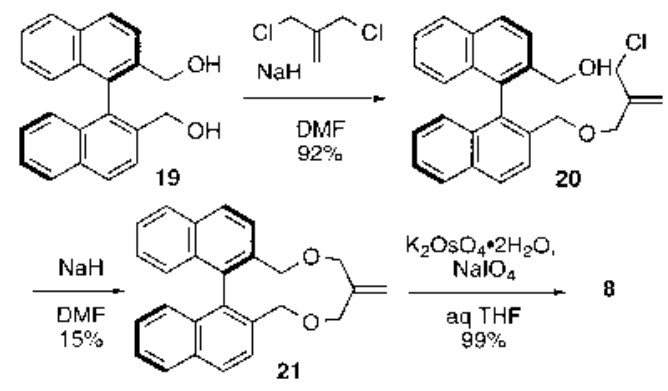

Chart 4. Synthesis of Chiral Ketone 8 from a Diol 19

Table 1. Asymmetric Epoxidation of Stilbene with Oxone ${ }^{\circledR}-$ Ketone $\mathbf{2}-\mathbf{8}^{a)}$

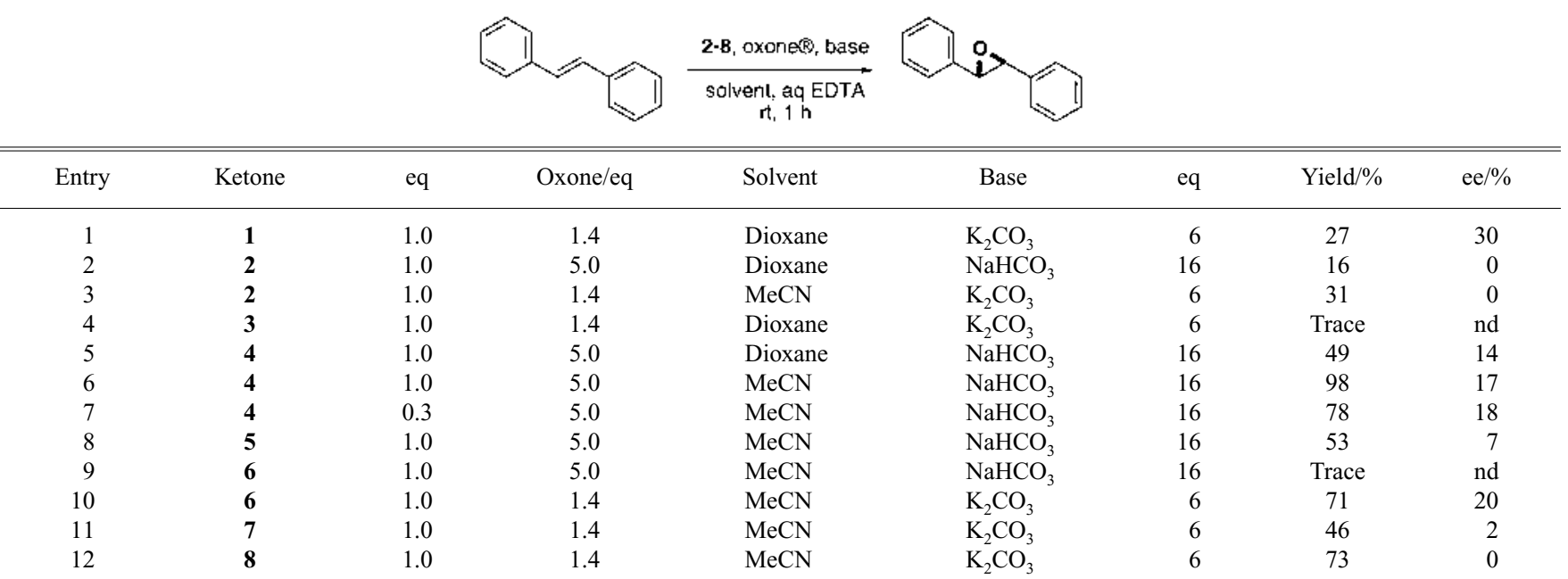

a) The data in entry 1 is quoted from ref. 7 for comparison purpose. Oxone ${ }^{\circledR}$ was added over a period of $1 \mathrm{~h}$ using a syringe drive, excepting $3 \mathrm{~h}$ for entry 10 . The mixture was then stirred at room temperature for another $1 \mathrm{~h}$, excepting $9 \mathrm{~h}$ for entry 1 . In entry 4 , acetonitrile could not be used because of insolubility of 4 . nd: not determined. 


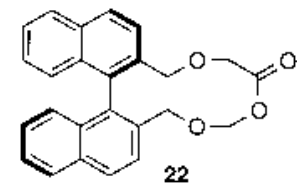

Fig. 3. Baeyer-Villiger Product 22

Villiger rearrangement occurred quite easily from the ketone 8 and gave a lactone $\mathbf{2 2}$ in 55\% chemical yield (entry 12).

Discussions on Catalytic Behaviour of the Cycloalkanones The asymmetric epoxidation with cycloalkanones 2 and $\mathbf{3}$ did not proceed smoothly, probably because of BaeyerVilliger type degradation of the ketone. This indicates that bulky arylsulfonylamide group is not effective for activation of dioxilane and prevention of Bayer-Villiger oxidative rearrangement. The situation is also same in the reactions using $\mathbf{7}$ and $\mathbf{8}$ though the relative epoxidation rate seemed to be faster than rearrangement. Hence, it is quite important to know that the ketones $\mathbf{4 - 6}$, especially trifluoromethanesulfonamide 4 in 0.3 eq, catalyzed the reaction giving the epoxide in the same enantioseletivity and $78 \%$ yield. Since the trifluoromethanesulfonyl group is highly electron-withdrawing in nature, the electrophilic reactivity of the ketone is significantly high in converting to a dioxilane, and the electronwithdrawing character of the amide retards Baeyer-Villiger oxidative rearrangement. These strongly indicated that electrophilic activation of ketone is possible by attaching electron-withdrawing sulfonylamide groups on both $\alpha$-methylene carbons of the ketone, but not by bulky sulfonylamide $(\mathbf{2}, \mathbf{3}$, 7) and ether group (8).

\section{Conclusion}

The chiral and $\mathrm{C} 2$ symmetric seven- and eleven-membered cycloalkanones $\mathbf{2}-\mathbf{8}$ bearing a 1,2-diphenylethane-1,2-diamine, cyclohexane-1,2-diamine, and binaphthalene backbones were prepared from the corresponding diamines $\mathbf{9 , 1 2}$, 16, and diol 19. Asymmetric epoxidation of stilbene with oxone $^{\circledR}$ was examined using these new cycloalkanones and was effectively catalyzed especially by the ketone 4 bearing trifluoromethanesulfonylamide group giving stilbene oxide in reasonably high 78 — $98 \%$ chemical yield and $18 \%$ ee.

\section{Experimental ${ }^{12)}$}

(2S,3S)-6-Methylene-2,3-diphenyl-1,4-bis(2,4,6-trimethylbenzenesulfonyl)[1,4]diazepane (11a) A solution of $\mathbf{1 0 a ^ { 8 }}(8.1 \mathrm{~g}, 14 \mathrm{mmol})$ in DMF $(24 \mathrm{ml})$ was added to a suspension of sodium hydride $(60 \%, 1.1 \mathrm{~g}, 28 \mathrm{mmol})$ in DMF $(4.0 \mathrm{ml})$ at $0^{\circ} \mathrm{C}$. The mixture was stirred at $80^{\circ} \mathrm{C}$ for $1 \mathrm{~h}$. To the mixture was added 3-iodo-2-iodomethylpropene $(5.2 \mathrm{~g}, 17 \mathrm{mmol})$ at $0^{\circ} \mathrm{C}$. The whole was stirred at $80^{\circ} \mathrm{C}$ for $1.5 \mathrm{~h}$ and was quenched with saturated (satd) $\mathrm{NH}_{4} \mathrm{Cl}$. The mixture was extracted with benzene $(100 \mathrm{ml})$. The combined organic layers were washed with $10 \% \mathrm{HCl}$, satd $\mathrm{NaHCO}_{3}$, and brine. Concentration and column chromatography $(\mathrm{AcOEt} /$ hexane $=1 / 5)$ gave 11a $(5.0 \mathrm{~g}, 58 \%)$ as a white amorphous of $[\alpha]_{\mathrm{D}}^{25}-162.1^{\circ}\left(c=1.51, \mathrm{CHCl}_{3}\right) .{ }^{1} \mathrm{H}-$ NMR: $1.93\left(6 \mathrm{H}, \mathrm{s}, \mathrm{CH}_{3}\right), 2.36\left(12 \mathrm{H}, \mathrm{s}, \mathrm{CH}_{3}\right), 4.65\left(2 \mathrm{H}, \mathrm{d}, J=16.8 \mathrm{~Hz}, \mathrm{CH}_{2}\right)$, $4.71\left(2 \mathrm{H}, \mathrm{d}, J=16.8 \mathrm{~Hz}, \mathrm{CH}_{2}\right), 5.15\left(2 \mathrm{H}, \mathrm{s}, \mathrm{CH}_{2}\right), 5.19\left(2 \mathrm{H}, \mathrm{s}, \mathrm{CH}_{2}\right), 6.50$ (4H, s, ArH), $6.58(4 \mathrm{H}, \mathrm{d}, J=7.3 \mathrm{~Hz}, \operatorname{ArH}), 6.72(4 \mathrm{H}, \mathrm{dd}, J=7.3,7.3 \mathrm{~Hz}$, ArH). $6.81(2 \mathrm{H}, \mathrm{d}, J=7.3 \mathrm{~Hz}, \mathrm{ArH}) .{ }^{13} \mathrm{C}-\mathrm{NMR}: 20.5,22.8,26.9,49.6,65.1$, $111.7,126.8,127.0,127.2,131.5,133.3,138.6,139.5,142.2$, 144.6. IR (Nujol): $1605,1470 \mathrm{~cm}^{-1}$. MS (FAB) $\mathrm{m} / z: 629\left(\mathrm{M}^{+}+\mathrm{H}\right)$. High resolution (HR)-MS $m / z$ : Calcd for $\mathrm{C}_{36} \mathrm{H}_{41} \mathrm{~N}_{2} \mathrm{O}_{4} \mathrm{~S}_{2}: 629.2508$. Found: 629.2512 .

(2S,3S)-2,3-Diphenyl-1,4-bis(2,4,6-trimethylbenzenesulfonyl)[1,4]diazepan-6-one (2) A solution of 11a $(4.1 \mathrm{~g}, 6.5 \mathrm{mmol})$ in chloroform (65 $\mathrm{ml}$ ) was ozonized at $-60^{\circ} \mathrm{C}$ by passing the $\mathrm{O}_{3} / \mathrm{O}_{2}$ stream until the solution was saturated with $\mathrm{O}_{3}$. Excess $\mathrm{O}_{3}$ was removed by $\mathrm{O}_{2}$ stream, and triph- enylphosphine (2.6 g, $9.7 \mathrm{mmol}$ ) was added portionwise to the reaction mixture. The whole was stirred at room temperature for $1 \mathrm{~h}$. Concentration and column chromatography $(\mathrm{AcOEt} / \mathrm{hexane}=1 / 1)$ gave $2(3.0 \mathrm{~g}, 92 \%)$ as colourless needles of mp $179.5-180.5^{\circ} \mathrm{C}$ (tert-butyl methyl ether (TBME)) and $[\alpha]_{\mathrm{D}}^{25}-5.9^{\circ}\left(c=1.52, \mathrm{CHCl}_{3}\right) .{ }^{1} \mathrm{H}-\mathrm{NMR}: 2.28\left(6 \mathrm{H}, \mathrm{s}, \mathrm{CH}_{3}\right), 2.31(12 \mathrm{H}$, s, $\left.\mathrm{CH}_{3}\right), 3.83\left(2 \mathrm{H}, \mathrm{d}, J=17.6 \mathrm{~Hz}, \mathrm{CH}_{2}\right), 4.69\left(2 \mathrm{H}, \mathrm{d}, J=17.6 \mathrm{~Hz}, \mathrm{CH}_{2}\right), 6.05$ $\left(2 \mathrm{H}, \mathrm{s}, \mathrm{CH}_{2}\right), 6.87\left(4 \mathrm{H}, \mathrm{s}, \mathrm{CH}_{2}\right), 7.16-7.17(4 \mathrm{H}, \mathrm{m}, \mathrm{ArH}) .7 .23-7.31(6 \mathrm{H}$, $\mathrm{m}, \mathrm{ArH}) .{ }^{13} \mathrm{C}-\mathrm{NMR}: 20.9,23.1,51.9,64.1,127.5,127.9,128.6,131.7$, 132.3, 138.0, 140.5, 143.1, 206.4. IR (Nujol): $1740,1430 \mathrm{~cm}^{-1}$. MS (FAB) $m / z: 632\left(\mathrm{M}^{+}+\mathrm{H}\right)$. HR-MS $m / z$ : Calcd for $\mathrm{C}_{35} \mathrm{H}_{39} \mathrm{~N}_{2} \mathrm{O}_{5} \mathrm{~S}_{2}: 631.2300$. Found: 631.2288 .

(2S,3S)-6-Methylene-2,3-diphenyl-1,4-bis(2,4,6-triisopropylbenzenesulfonyl)[1,4]diazepane (11b) Prepared from $10 b^{8)}$ and 3-iodo-2iodomethylpropene, according to the procedure for 11a. Column chromatography $(\mathrm{AcOEt} / \mathrm{hexane}=1 / 10)$ gave $11 \mathrm{~b}$ in $66 \%$ yield as a white amorphous of $[\alpha]_{\mathrm{D}}^{25}-50.4^{\circ}\left(c=1.10, \mathrm{CHCl}_{3}\right) .{ }^{1} \mathrm{H}-\mathrm{NMR}: 1.11\left(24 \mathrm{H}, \mathrm{d}, J=5.2 \mathrm{~Hz}, \mathrm{CH}_{3}\right.$ $2.82(2 \mathrm{H}$, seqt, $J=6.9 \mathrm{~Hz}, \mathrm{CH}), 3.75(4 \mathrm{H}$, seqt, $J=6.9 \mathrm{~Hz}, \mathrm{CH}), 4.28(2 \mathrm{H}, \mathrm{d}$, $\left.J=16.7 \mathrm{~Hz}, \mathrm{CH}_{2}\right), 4.70\left(2 \mathrm{H}, \mathrm{d}, J=16.7 \mathrm{~Hz}, \mathrm{CH}_{2}\right), 5.02\left(2 \mathrm{H}, \mathrm{s}, \mathrm{CH}_{2}\right), 5.62$ $\left(2 \mathrm{H}, \mathrm{s}, \mathrm{CH}_{2}\right), 7.00-7.10(14 \mathrm{H}, \mathrm{m}, \mathrm{ArH}) .{ }^{13} \mathrm{C}-\mathrm{NMR}: 23.50,23.53,24.75$, $24.8,29.5,34.1,48.2,64.5,123.8,127.4,127.9,128.2,131.8,139.9,145.6$, 151.4, 153.1. IR (Nujol): $1600,1460 \mathrm{~cm}^{-1}$. MS (FAB) $m / z: 797\left(\mathrm{M}^{+}+\mathrm{H}\right)$. HR-MS $m / z$ : Calcd for $\mathrm{C}_{48} \mathrm{H}_{65} \mathrm{~N}_{2} \mathrm{O}_{4} \mathrm{~S}_{2}: 797.4386$. Found: 797.4393.

(2S,3S)-2,3-Diphenyl-1,4-bis(2,4,6-triisopropylbenzenesulfonyl)[1,4]diazepan-6-one (3) Prepared from 11b, according to the procedure for $\mathbf{2}$. Column chromatography (TBME/hexane $=1 / 10$ ) gave $\mathbf{3}$ in $80 \%$ yield as a white powder of $\mathrm{mp} 198.5-200.5^{\circ} \mathrm{C}$ and $[\alpha]_{\mathrm{D}}^{25}+18.9^{\circ}\left(c=0.54, \mathrm{CHCl}_{3}\right)$. ${ }^{1} \mathrm{H}-\mathrm{NMR}$ : $0.96\left(12 \mathrm{H}, \mathrm{d}, J=6.7 \mathrm{~Hz}, \mathrm{CH}_{3}\right), 1.06\left(12 \mathrm{H}, \mathrm{d}, J=6.7 \mathrm{~Hz}, \mathrm{CH}_{3}\right), 1.24$ $\left(12 \mathrm{H}, \mathrm{d}, J=7.0 \mathrm{~Hz}, \mathrm{CH}_{3}\right), 2.88(2 \mathrm{H}$, seqt, $J=6.7 \mathrm{~Hz}, \mathrm{CH}), 3.62(4 \mathrm{H}$, seqt, $J=6.7 \mathrm{~Hz}, \mathrm{CH}), 3.88\left(2 \mathrm{H}, \mathrm{d}, J=17.7 \mathrm{~Hz}, \mathrm{CH}_{2}\right), 4.81(2 \mathrm{H}, \mathrm{d}, J=17.7 \mathrm{~Hz}$, $\left.\mathrm{CH}_{2}\right), 6.09\left(2 \mathrm{H}, \mathrm{s}, \mathrm{CH}_{2}\right), 7.11(4 \mathrm{H}, \mathrm{s}, \mathrm{ArH}), 7.26-7.35(10 \mathrm{H}, \mathrm{m}, \mathrm{ArH}) .{ }^{13} \mathrm{C}-$ NMR: $23.50,23.53,24.75,24.78,29.7,34.1,51.4,63.4,124.3,127.8$, $127.9,128.9,130.3,138.9,151.9,153.7,206.4$. IR (Nujol): $1740 \mathrm{~cm}^{-1}$. MS (FAB) $m / z$ : $799\left(\mathrm{M}^{+}+\mathrm{H}\right)$. HR-MS $m / z$ : Calcd for $\mathrm{C}_{47} \mathrm{H}_{63} \mathrm{~N}_{2} \mathrm{O}_{5} \mathrm{~S}_{2}$ : 799.4178 . Found: 799.4189 .

$(6 R, 7 R)$-3-Methylene-1,5-bistrifluoromethanesulfonyldecahydrobenzo$[b][1,4]$ diazepine $\left(14: \mathbf{R}=\mathrm{CF}_{3} \mathrm{SO}_{2}\right)$ Prepared from $13\left(\mathrm{R}=\mathrm{CF}_{3} \mathrm{SO}_{2}\right)^{13)}$ and 3-iodo-2-iodomethylpropene, according to the procedure for 11. Column chromatography $(\mathrm{AcOEt} /$ hexane $=1 / 5)$ and recrystallization gave $\mathbf{1 4}$ $\left(\mathrm{CF}_{3} \mathrm{SO}_{2}\right)$ in $61 \%$ yield as a white powder of $\mathrm{mp} 145-146^{\circ} \mathrm{C}$ (diethyl ether) and $[\alpha]_{\mathrm{D}}^{25}-12.3^{\circ}\left(c=1.30, \mathrm{CHCl}_{3}\right)$. ${ }^{1} \mathrm{H}-\mathrm{NMR}: 1.33-1.42\left(2 \mathrm{H}, \mathrm{m}, \mathrm{CH}_{2}\right)$, $1.80-2.11\left(6 \mathrm{H}, \mathrm{m}, \mathrm{CH}_{2}\right), 3.83(2 \mathrm{H}, \mathrm{brs}, \mathrm{CH}), 4.17-4.28\left(4 \mathrm{H}, \mathrm{m}, \mathrm{CH}_{2}\right)$, $5.15\left(2 \mathrm{H}, \mathrm{s}, \mathrm{CH}_{2}\right) .{ }^{13} \mathrm{C}$-NMR: 25.0, 31.1, 65.9, 66.1, $119.3(\mathrm{q}, J=321 \mathrm{~Hz})$, 119.8, 137.4. IR (Nujol): $3400,1180 \mathrm{~cm}^{-1}$. MS (FAB) $m / z: 431\left(\mathrm{M}^{+}+\mathrm{H}\right)$. Anal. Calcd for $\mathrm{C}_{12} \mathrm{H}_{16} \mathrm{~F}_{6} \mathrm{~N}_{2} \mathrm{O}_{4} \mathrm{~S}_{2}: \mathrm{C}, 33.49 ; \mathrm{H}, 3.75 ; \mathrm{N}, 6.51$. Found: $\mathrm{C}$, $33.37 ; \mathrm{H}, 3.71 ; \mathrm{N}, 6.53$.

$(6 R, 7 R)-1,5$-Bistrifluoromethanesulfonyldecahydrobenzo $[b][1,4] \mathrm{di}-$ azepin-3-one (4) Prepared from $14\left(\mathrm{R}=\mathrm{CF}_{3} \mathrm{SO}_{2}\right)$, according to the procedure for 2 . Column chromatography $(\mathrm{AcOEt} /$ hexane $=1 / 1)$ and recrystallization gave 4 in $76 \%$ yield as colourless granules of $\mathrm{mp} 126.5^{\circ} \mathrm{C}$ (dec.) $\left(\mathrm{CHCl}_{3}-\right.$ hexane $)$ and $[\alpha]_{\mathrm{D}}^{25}-23.9^{\circ}(c=1.76, \mathrm{MeOH}) .{ }^{1} \mathrm{H}-\mathrm{NMR}: 1.39-2.14$ $\left(8 \mathrm{H}, \mathrm{m}, \mathrm{CH}_{2}\right), 3.78(2 \mathrm{H}, \mathrm{m}, \mathrm{CH}), 4.21\left(2 \mathrm{H}, \mathrm{d}, J=18.5 \mathrm{~Hz}, \mathrm{CH}_{2}\right), 4.44(2 \mathrm{H}, \mathrm{d}$, $\left.J=18.5 \mathrm{~Hz}, \mathrm{CH}_{2}\right) .{ }^{13} \mathrm{C}-\mathrm{NMR}: 24.7,29.5,59.4,65.4,119.3(\mathrm{q}, J=323 \mathrm{~Hz})$, 199.3. IR (Nujol): $1725 \mathrm{~cm}^{-1}$. MS (FAB) $m / z$ : $433\left(\mathrm{M}^{+}+\mathrm{H}\right)$. Anal. Calcd for $\mathrm{C}_{11} \mathrm{H}_{14} \mathrm{~F}_{6} \mathrm{~N}_{2} \mathrm{O}_{5} \mathrm{~S}_{2}: \mathrm{C}, 30.56 ; \mathrm{H}, 3.26$ Found: $\mathrm{C}, 30.48 ; \mathrm{H}, 3.27$.

$(6 R, 7 R)-1,5$-Bisbenzenesulfonyl-3-methylenedecahydrobenzo $[b][1,4]-$ diazepine (14: $\left.\mathbf{R}=\mathrm{PhSO}_{2}\right)$ Prepared from $\mathbf{1 3}\left(\mathrm{R}=\mathrm{PhSO}_{2}\right)$ and 3-iodo-2iodomethylpropene, according to the procedure for 11a. Column chromatography $(\mathrm{AcOEt} /$ hexane $=1 / 2)$ and recrystallization gave $14\left(\mathrm{R}=\mathrm{PhSO}_{2}\right)$ in $84 \%$ yield as colorless cubes of $\mathrm{mp} 165-166^{\circ} \mathrm{C}$ (benzene) and $[\alpha]_{\mathrm{D}}^{25}$ $+18.7^{\circ}\left(c=1.68, \mathrm{CHCl}_{3}\right) .{ }^{1} \mathrm{H}-\mathrm{NMR}: 1.25\left(2 \mathrm{H}, \mathrm{m}, \mathrm{CH}_{2}\right), 1.73\left(4 \mathrm{H}, \mathrm{m}, \mathrm{CH}_{2}\right)$, $1.93\left(2 \mathrm{H}, \mathrm{m}, \mathrm{CH}_{2}\right), 3.57(2 \mathrm{H}, \mathrm{m}, \mathrm{CH}), 3.99\left(2 \mathrm{H}, \mathrm{d}, J=15.6 \mathrm{~Hz}, \mathrm{CH}_{2}\right), 4.07$ $\left(2 \mathrm{H}, \mathrm{d}, J=15.6 \mathrm{~Hz}, \mathrm{CH}_{2}\right), 4.90\left(2 \mathrm{H}, \mathrm{s}, \mathrm{CH}_{2}\right), 7.44-7.52(6 \mathrm{H}, \mathrm{m}, \mathrm{ArH})$, 7.83-7.86 (4H, m, ArH). ${ }^{13} \mathrm{C}-\mathrm{NMR}: 25.4,31.3,52.0,64.1,116.5,127.1$, 128.8, 132.1, 139.8, 142.3. IR (Nujol): $1650,1320 \mathrm{~cm}^{-1}$. MS (FAB) $\mathrm{m} / \mathrm{z}$ : $447\left(\mathrm{M}^{+}+\mathrm{H}\right)$. Anal. Calcd for $\mathrm{C}_{22} \mathrm{H}_{26} \mathrm{~N}_{2} \mathrm{O}_{4} \mathrm{~S}_{2}: \mathrm{C}, 59.17 ; \mathrm{H}, 5.87 ; \mathrm{N}, 6.27$. Found: C, 59.07; H, 5.86; N, 6.11.

$(6 R, 7 R) 1,5$-Bisbenzenesulfonyldecahydrobenzo $[b][1,4]$ diazepin-3-one (5) Prepared from $14\left(\mathrm{R}=\mathrm{PhSO}_{2}\right)$ according to the procedure for 2 . Column chromatography (AcOEt/hexane $=1 / 2)$ and recrystallization gave $\mathbf{5}$ in $99 \%$ yield as colourless needles of $\mathrm{mp} 195-196^{\circ} \mathrm{C}$ (benzene-hexane) and $[\alpha]_{\mathrm{D}}^{25}-26.2^{\circ}\left(c=1.55, \mathrm{CHCl}_{3}\right) .{ }^{1} \mathrm{H}-\mathrm{NMR}: 1.21-1.30\left(2 \mathrm{H}, \mathrm{m}, \mathrm{CH}_{2}\right), 1.74-$ $1.83\left(6 \mathrm{H}, \mathrm{m}, \mathrm{CH}_{2}\right), 3.66-3.68(2 \mathrm{H}, \mathrm{m}, \mathrm{CH}), 4.15\left(2 \mathrm{H}, \mathrm{d}, J=18.3 \mathrm{~Hz}, \mathrm{CH}_{2}\right)$, $4.21\left(2 \mathrm{H}, \mathrm{d}, J=18.3 \mathrm{~Hz}, \mathrm{CH}_{2}\right), 7.49-7.52(4 \mathrm{H}, \mathrm{m}, \mathrm{ArH}), 7.55-7.58(2 \mathrm{H}$, $\mathrm{m}, \mathrm{ArH}), 7.82-7.84(4 \mathrm{H}, \mathrm{m}, \mathrm{ArH}) .{ }^{13} \mathrm{C}-\mathrm{NMR}: 25.0,29.9,56.3,63.9,126.7$, 
129.2, 132.7, 141.6, 204.2. IR (Nujol): $1720 \mathrm{~cm}^{-1}$. MS (FAB) $\mathrm{m} / z: 449$ $\left(\mathrm{M}^{+}+\mathrm{H}\right)$. Anal. Calcd for $\mathrm{C}_{21} \mathrm{H}_{24} \mathrm{~N}_{2} \mathrm{O}_{5} \mathrm{~S}_{2}$ : C, 56.23; H, 5.39. Found: $\mathrm{C}$, $56.04 ; \mathrm{H}, 5.36$.

$(1 R, 2 R)-N, N^{\prime}$-Bis $\left(5,5\right.$-dimethyl-2-oxo-2 $\lambda^{5}-[1,3,2]$ dioxaphosphinan-2yl)cyclohexane-1,2-diamine (13: $\mathrm{R}=\mathrm{PO}\left(\mathrm{OCH}_{2} \mathrm{C}(\mathrm{Me})_{2} \mathrm{CH}_{2} \mathrm{O}\right)$ ) A solution of 2-chloro-5,5-dimethyl[1,3,2]dioxaphosphinane 2-oxide (11 g, 57 mmol) in $\mathrm{CH}_{2} \mathrm{Cl}_{2}(60 \mathrm{ml})$ was added to a solution of 12 in $\mathrm{CH}_{2} \mathrm{Cl}_{2}(24 \mathrm{ml})$ containing $N, N$-diisopropylethylamine $(20 \mathrm{ml}, 110 \mathrm{mmol})$ at $0{ }^{\circ} \mathrm{C}$. The mixture was stirred at room temperature for $12 \mathrm{~h}$, and was poured onto $10 \% \mathrm{HCl}$ $(100 \mathrm{ml})$, and then extracted with $\mathrm{CHCl}_{3}(100 \mathrm{ml})$. The combined organic layers were washed with satd $\mathrm{NaHCO}_{3}$, brine. Concentration and recrystallization from AcOEt-CHCl $\mathrm{CH}_{3}$ afforded $13\left(\mathrm{R}=\mathrm{PO}\left(\mathrm{OCH}_{2} \mathrm{C}(\mathrm{Me})_{2} \mathrm{CH}_{2} \mathrm{O}\right)\right)$ in $64 \%$ yield as a white powder of $\mathrm{mp} 185-188^{\circ} \mathrm{C}$ and $[\alpha]_{\mathrm{D}}^{25}+8.3^{\circ}(c=1.44$, $\mathrm{CHCl}_{3}$ ). ${ }^{1} \mathrm{H}-\mathrm{NMR}: 1.01,1.09$ (each $\left.6 \mathrm{H}, \mathrm{s}, \mathrm{CH}_{3}\right), 1.26\left(4 \mathrm{H}, \mathrm{m}, \mathrm{CH}_{2}\right), 1.69$, $2.17\left(\right.$ each $\left.2 \mathrm{H}, \mathrm{m}, \mathrm{CH}_{2}\right), 2.86(2 \mathrm{H}, \mathrm{m}, \mathrm{CH}), 3.51(2 \mathrm{H}, \mathrm{m}, \mathrm{NH}), 3.91(1 \mathrm{H}, \mathrm{dd}$, $\left.J=11.6,11.6 \mathrm{~Hz}, \mathrm{CH}_{2}\right), 3.91\left(1 \mathrm{H}, \mathrm{dd}, J=10.1,10.1 \mathrm{~Hz}, \mathrm{CH}_{2}\right), 3.94(1 \mathrm{H}, \mathrm{dd}$, $\left.J=11.6,11.6 \mathrm{~Hz}, \mathrm{CH}_{2}\right), 3.94\left(1 \mathrm{H}, \mathrm{dd}, J=10.1,10.1 \mathrm{~Hz}, \mathrm{CH}_{2}\right), 4.13(2 \mathrm{H}, \mathrm{dd}$, $\left.J=10.1,10.1 \mathrm{~Hz}, \mathrm{CH}_{2}\right), 4.14\left(2 \mathrm{H}\right.$, dd, $\left.J=10.1,10.1 \mathrm{~Hz}, \mathrm{CH}_{2}\right) \cdot{ }^{13} \mathrm{C}-\mathrm{NMR}$ $\left(\mathrm{CDCl}_{3}\right): 21.1,21.5,24.7,32.0(\mathrm{~d}, J=5.2 \mathrm{~Hz}), 34.7,56.4(J=7.2 \mathrm{~Hz}), 76.3$ (d, $J=6.2 \mathrm{~Hz}$ ), 76.5 (d, $J=6.2 \mathrm{~Hz}$ ). IR (Nujol): $3200,1275 \mathrm{~cm}^{-1}$. MS $\mathrm{m} / z$ : $410\left(\mathrm{M}^{+}\right)$. HR-MS $\mathrm{m} / \mathrm{z}$ : Calcd for $\mathrm{C}_{16} \mathrm{H}_{32} \mathrm{~N}_{2} \mathrm{O}_{6} \mathrm{P}_{2}$ : 410.1736. Found: 410.1733 .

$(6 R, 7 R)-1,5-B i s\left(5,5-d i m e t h y l-2-0 x 0-\lambda^{5}-[1,3,2]\right.$ dioxaphosphin-2-yl)decahydrobenzo[b] $[1,4]$ diazepin-3-one (6) Prepared from 13 ( $\mathrm{R}=\mathrm{PO}-$ $\left.\left(\mathrm{OCH}_{2} \mathrm{C}(\mathrm{Me})_{2} \mathrm{CH}_{2} \mathrm{O}\right)\right)$, according to the procedure for 11a and 2. Column chromatography $(\mathrm{EtOH} / \mathrm{AcOEt}=1 / 20)$ and recrystallization gave 6 in $48 \%$ two-step yield as pale yellow granules of $\mathrm{mp} 155-156^{\circ} \mathrm{C}$ (AcOEt) and $[\alpha]_{\mathrm{D}}^{25}+17.2^{\circ}\left(c=1.33, \mathrm{CHCl}_{3}\right) .{ }^{1} \mathrm{H}-\mathrm{NMR}: 0.93,1.18\left(\right.$ each $\left.6 \mathrm{H}, \mathrm{s}, \mathrm{CH}_{3}\right)$, $1.34,1.78$ (each $\left.2 \mathrm{H}, \mathrm{m}, \mathrm{CH}_{2}\right), 1.88\left(2 \mathrm{H}, \mathrm{m}, \mathrm{CH}_{2}\right), 2.00\left(2 \mathrm{H}, \mathrm{m}, \mathrm{CH}_{2}\right), 3.43$ $(2 \mathrm{H}, \mathrm{m}, \mathrm{CH}), 3.81\left(2 \mathrm{H}, \mathrm{dd}, J=7.7,18.0 \mathrm{~Hz}, \mathrm{CH}_{2}\right), 3.96(2 \mathrm{H}, \mathrm{dd}, J=18.3$, $\left.18.3 \mathrm{~Hz}, \mathrm{CH}_{2}\right), 3.98\left(2 \mathrm{H}, \mathrm{dd}, J=18.3,18.3 \mathrm{~Hz}, \mathrm{CH}_{2}\right), 4.03(2 \mathrm{H}, \mathrm{dd}, J=10.7$, $\left.18.0 \mathrm{~Hz}, \mathrm{CH}_{2}\right), 4.19\left(2 \mathrm{H}, \mathrm{dd}, J=11.0,11.0 \mathrm{~Hz}, \mathrm{CH}_{2}\right), 4.20(2 \mathrm{H}, \mathrm{dd}, J=11.0$, $\left.11.0 \mathrm{~Hz}, \mathrm{CH}_{2}\right) .{ }^{13} \mathrm{C}$-NMR: $21.0,21.9,25.3,30.6,31.8(\mathrm{~d}, J=5.2 \mathrm{~Hz}), 55.6$, 62.5, 76.3 (d, $J=6.3 \mathrm{~Hz}), 76.4$ (d, $J=6.3 \mathrm{~Hz}), 206.4$. IR (Nujol): $1715 \mathrm{~cm}^{-1}$. MS $m / z$ : $464\left(\mathrm{M}^{+}\right)$. HR-MS (FAB) $m / z$ : Calcd for $\mathrm{C}_{19} \mathrm{H}_{35} \mathrm{~N}_{2} \mathrm{O}_{7} \mathrm{P}_{2}: 465.1919$. Found: 465.1910 .

(R)-[1,1']Binaphthalenyl-2,2'-dicarboxylic Acid Diamide (16a) A solution of $(R)-15(1.69 \mathrm{~g}, 4.94 \mathrm{mmol})$ in thionyl chloride $(5.0 \mathrm{ml})$ was refluxed with stirring for $1 \mathrm{~h}$. After the whole was concentrated, benzene $(5.0 \mathrm{ml})$ and aqueous $\mathrm{NH}_{3}(28 \%, 5.0 \mathrm{ml})$ were added. The mixture was stirred at room temperature for $0.5 \mathrm{~h}$, and then poured onto $10 \% \mathrm{HCl}$, and was extracted with AcOEt $(100 \mathrm{ml})$. The organic layer was washed with brine. Concentration, column chromatography (hexane/AcOEt $=9 / 1$, then $\mathrm{AcOEt}$ ), and recrystallization gave $16 \mathrm{a}(1.53 \mathrm{~g}, 91 \%)$ as colourless cubes of $\mathrm{mp} 222.5-$ $224.0^{\circ} \mathrm{C}$ (benzene) and $[\alpha]_{\mathrm{D}}^{25}+237.5^{\circ}\left(c=0.80, \mathrm{CHCl}_{3}\right) .{ }^{1} \mathrm{H}-\mathrm{NMR}: 5.44$ $\left(2 \mathrm{H}\right.$, br s, $\left.\mathrm{NH}_{2}\right), 7.04\left(2 \mathrm{H}\right.$, br s, $\left.\mathrm{NH}_{2}\right), 7.11(2 \mathrm{H}, \mathrm{d}, J=8.6 \mathrm{~Hz}, \mathrm{ArH}), 7.25-$ $7.28(2 \mathrm{H}, \mathrm{m}, \mathrm{ArH}), 7.47-7.50(2 \mathrm{H}, \mathrm{m}, \mathrm{ArH}), 7.73(2 \mathrm{H}, \mathrm{d}, J=8.6 \mathrm{~Hz}, \mathrm{ArH})$, $7.92(2 \mathrm{H}, \mathrm{d}, J=8.3 \mathrm{~Hz}, \mathrm{ArH}), 8.01(2 \mathrm{H}, \mathrm{d}, J=8.3 \mathrm{~Hz}, \mathrm{ArH}) .{ }^{13} \mathrm{C}-\mathrm{NMR}$ : 123.7, 126.4, 127.2, 127.3, 128.2, 128.3, 129.0, 132.3, 133.1, 134.2, 172.4 . IR (Nujol): $3300,3150,1660 \mathrm{~cm}^{-1}$. MS m/z: $340\left(\mathrm{M}^{+}\right)$. HR-MS $\mathrm{m} / \mathrm{z}$ : Calcd for $\mathrm{C}_{22} \mathrm{H}_{16} \mathrm{~N}_{2} \mathrm{O}_{2}: 340.1212$. Found: 340.1217 .

(R)-N, $N^{\prime}$-Bisbenzenesulfonyl-1,1'-binaphthyl-2,2'-bis(methylamine) (17) A solution of $16 \mathrm{a}(525 \mathrm{mg}, 1.54 \mathrm{mmol})$ in tetrahydrofuran (THF) $(2.5 \mathrm{ml})$ was added to $\mathrm{BH}_{3} \cdot \mathrm{Me}_{2} \mathrm{~S}$ in THF $(2.5 \mathrm{ml}, 10 \mathrm{M})$ and refluxed for $2 \mathrm{~d}$ with stirring. To the mixture were added $\mathrm{MeOH}(8.0 \mathrm{ml})$ and aqueous $\mathrm{HCl}$ $(35 \%, 1.0 \mathrm{ml})$ at $0{ }^{\circ} \mathrm{C}$ and then the mixture was concentrated. The residue was poured onto $15 \% \mathrm{NaOH}(15 \mathrm{ml})$ and extracted with TBME $(30 \mathrm{ml})$. Combined organic layers were washed with satd $\mathrm{NaHCO}_{3}(15 \mathrm{ml})$ and brine. Concentration gave $(R)-16 \mathbf{b}(360 \mathrm{mg}, 75 \%)$ as a pale yellow oil, which was used in the next step without any purification.

To a solution of $16 \mathbf{b}(100 \mathrm{mg}, 0.29 \mathrm{mmol})$ in $\mathrm{CH}_{2} \mathrm{Cl}_{2}(3.0 \mathrm{ml})$ containing triethylamine (TEA, $0.12 \mathrm{ml}, 0.87 \mathrm{mmol}$ ) was added benzenesulfonyl chloride $(0.09 \mathrm{ml}, 0.73 \mathrm{mmol})$ at $0{ }^{\circ} \mathrm{C}$. The whole was stirred for $1 \mathrm{~h}$ at room temperature. After addition of water $(10 \mathrm{ml})$, the mixture was extracted with benzene $(30 \mathrm{ml})$. The extract was washed with $10 \% \mathrm{HCl}$, satd $\mathrm{NaHCO}_{3}$ and brine. Concentration and column chromatography (AcOEt/hexane $=1 / 2$ ) gave $17(150 \mathrm{mg}, 89 \%)$ as a white amorphous of $\mathrm{mp} 85-89^{\circ} \mathrm{C}$ and $[\alpha]_{\mathrm{D}}^{25}$ $+86.9^{\circ}\left(c=0.72, \mathrm{CHCl}_{3}\right) .{ }^{1} \mathrm{H}-\mathrm{NMR}: 3.68,3.73$ (each $2 \mathrm{H}, \mathrm{dd}, J=6.0$, $\left.14.4 \mathrm{~Hz}, \mathrm{CH}_{2}\right), 4.69(2 \mathrm{H}, \mathrm{dd}, J=6.0,6.0 \mathrm{~Hz}, \mathrm{NH}), 6.92(2 \mathrm{H}, \mathrm{d}, J=8.6 \mathrm{~Hz}$, $\mathrm{ArH}), 7.24-7.26(2 \mathrm{H}, \mathrm{m}, \mathrm{ArH}), 7.32-7.36(6 \mathrm{H}, \mathrm{m}, \mathrm{ArH}), 7.46-7.49(4 \mathrm{H}$, $\mathrm{m}, \operatorname{ArH}), 7.52(2 \mathrm{H}, \mathrm{d}, J=8.0 \mathrm{~Hz}, \mathrm{ArH}), 7.64(2 \mathrm{H}, \mathrm{d}, J=8.6 \mathrm{~Hz}, \mathrm{ArH}), 7.92$ $(4 \mathrm{H}, \mathrm{dd}, J=8.6,8.6 \mathrm{~Hz}, \mathrm{ArH}) .{ }^{13} \mathrm{C}-\mathrm{NMR}\left(\mathrm{CDCl}_{3}\right): 45.1,125.5,126.4,126.5$, $126.7,127.1,128.31,128.33,129.0,132.51,132.53,133.0,133.1,133.6$, 139.3. IR (Nujol): $3250,1325 \mathrm{~cm}^{-1}$. MS (FAB) $m / z: 593\left(\mathrm{M}^{+}+\mathrm{H}\right)$. HR-MS $m / z$ : Calcd for $\mathrm{C}_{34} \mathrm{H}_{29} \mathrm{~N}_{2} \mathrm{O}_{4} \mathrm{~S}_{2}: 593.1569$. Found: 593.1577.

(R)-10-Methylene-8,12-bis(phenylsulfonyl)-8,9,10,11,12,13-hexahydro7H-dinaphtho[2,1-g:1,2-i][1,5]diazacycloundecine (18) Prepared from 17 and 3-iodo-2-iodomethylpropenein, according to the procedure for 11a. Recrystallization gave $\mathbf{1 8}$ in $\mathbf{7 2 \%}$ yield as colourless needles of $\mathrm{mp} 203.5-$ $205^{\circ} \mathrm{C}(\mathrm{AcOEt})$ and $[\alpha]_{\mathrm{D}}^{25}+182^{\circ}\left(c=0.98, \mathrm{CHCl}_{3}\right) .{ }^{1} \mathrm{H}-\mathrm{NMR}: 3.38(2 \mathrm{H}, \mathrm{d}$, $\left.J=15.9 \mathrm{~Hz}, \mathrm{CH}_{2}\right), 3.53\left(2 \mathrm{H}, \mathrm{d}, J=14.7 \mathrm{~Hz}, \mathrm{CH}_{2}\right), 3.55(2 \mathrm{H}, \mathrm{d}, J=15.9 \mathrm{~Hz}$, $\left.\mathrm{CH}_{2}\right), 4.44\left(2 \mathrm{H}, \mathrm{d}, J=14.7 \mathrm{~Hz}, \mathrm{CH}_{2}\right), 4.65\left(2 \mathrm{H}, \mathrm{s}, \mathrm{CH}_{2}\right), 7.01(2 \mathrm{H}, \mathrm{d}, J=8.6$ $\mathrm{Hz}, \mathrm{ArH}) .7 .25-7.30(2 \mathrm{H}, \mathrm{m}, \mathrm{ArH}), 7.43-7.49(6 \mathrm{H}, \mathrm{m}, \mathrm{ArH}), 7.52-7.55$ (2H, m, ArH), 7.68-7.73 (6H, m, ArH) $7.90-7.94(4 \mathrm{H}, \mathrm{m}, \mathrm{ArH}) .{ }^{13} \mathrm{C}-$ NMR: $49.3,50.8,118.5,125.9,126.3,126.7,127.0,127.1,128.2,129.0$, $132.7,132.9,133.0,133.1,134.2,136.3,138.9$. IR (Nujol): $1640 \mathrm{~cm}^{-1}$. MS (FAB) $m / z$ : $645\left(\mathrm{M}^{+}+\mathrm{H}\right)$. HR-MS $m / z$ : Calcd for $\mathrm{C}_{38} \mathrm{H}_{33} \mathrm{~N}_{2} \mathrm{O}_{4} \mathrm{~S}_{2}: 645.1882$. Found: 645.1901 .

(R)-8,12-Bis(phenylsulfonyl)-7,8,9,11,12,13-hexahydro-10H-dinaphtho[2,1-g:1,2-i][1,5]diazacycloundecine-10-one (7) To a suspension of $18(64 \mathrm{mg}, 0.1 \mathrm{mmol})$ and $\mathrm{K}_{2} \mathrm{OsO}_{4} \cdot 2 \mathrm{H}_{2} \mathrm{O}(3 \mathrm{mg}, 0.01 \mathrm{mmol}$ in THF $(1.5 \mathrm{ml})$ was added a solution of sodium metaperiodate $(63 \mathrm{mg}, 0.3 \mathrm{mmol})$ in water $(0.5 \mathrm{ml})$. The whole was stirred at room temperature for $2.5 \mathrm{~d}$. After addition of water $(10 \mathrm{ml})$, the mixture was extracted with AcOEt. The extract was washed with $10 \% \mathrm{HCl}$, satd $\mathrm{NaHCO}_{3}$ and brine. Concentration and column chromatography (benzene) gave $7(38 \mathrm{mg}, 59 \%)$ as a solid of $\mathrm{mp} 146-$ $148^{\circ} \mathrm{C}$ and $[\alpha]_{\mathrm{D}}^{25}+216.6^{\circ}\left(c=0.27, \mathrm{CHCl}_{3}\right) .{ }^{1} \mathrm{H}-\mathrm{NMR}: 3.24(2 \mathrm{H}, \mathrm{d}, J=16.2$ $\left.\mathrm{Hz}, \mathrm{CH}_{2}\right), 3.57\left(2 \mathrm{H}, \mathrm{d}, J=13.2 \mathrm{~Hz}, \mathrm{CH}_{2}\right), 4.05\left(2 \mathrm{H}, \mathrm{d}, J=16.2 \mathrm{~Hz}, \mathrm{CH}_{2}\right)$, $4.30\left(2 \mathrm{H}, \mathrm{d}, J=13.2 \mathrm{~Hz}, \mathrm{CH}_{2}\right), 7.25(2 \mathrm{H}, \mathrm{d}, J=8.3 \mathrm{~Hz}, \mathrm{ArH}) .7 .36-7.39$ (4H, m, ArH), 7.46-7.49 (4H, m, ArH), 7.54-7.59 (8H, m, ArH), 7.99$7.03(4 \mathrm{H}, \mathrm{m}, \mathrm{ArH}) .{ }^{13} \mathrm{C}-\mathrm{NMR} \delta: 52.6,53.9,125.3,126.6,127.1,127.9$, 128.3, 128.4, 128.6, 129.1, 130.5, 132.6, 133.1, 134.2, 135.2, 136.2, 201.1. IR (Nujol): $1735 \mathrm{~cm}^{-1}$. MS (FAB) $m / z: 647\left(\mathrm{M}^{+}+\mathrm{H}\right)$. HR-MS $m / z$ : Calcd for $\mathrm{C}_{37} \mathrm{H}_{31} \mathrm{~N}_{2} \mathrm{O}_{5} \mathrm{~S}_{2}: 647.1674$. Found: 647.1668 .

(R)-[2'-(2-Chloromethylallyloxymethyl) [1, $\left.1^{\prime}\right]$ binaphthalenyl-2yl]methanol (20) A solution of a diol $19(443 \mathrm{mg}, 1.41 \mathrm{mmol})$ in DMF $(11 \mathrm{ml})$ was added to a suspension of sodium hydride $(60 \%, 57 \mathrm{mg}, 1.43$ $\mathrm{mmol})$ in DMF $(130 \mathrm{ml})$ at $0{ }^{\circ} \mathrm{C}$. The mixture was stirred at room temperature for $1 \mathrm{~h}$. To the mixture was added 3-chloro-2-chloromethylpropene $(4.90 \mathrm{ml}, 42.3 \mathrm{mmol})$ at $-40^{\circ} \mathrm{C}$. The whole was stirred at $-40^{\circ} \mathrm{C}$ for $24 \mathrm{~h}$ and was quenched with satd $\mathrm{NH}_{4} \mathrm{Cl}$. The mixture was extracted with benzene $(30 \mathrm{ml})$. The combined organic layers were washed with brine. Concentration and column chromatography (AcOEt/hexane $=1 / 6)$ gave $20(520 \mathrm{mg}$, $92 \%)$ as a colourless gum of $[\alpha]_{\mathrm{D}}^{25}+104.4^{\circ}\left(c=0.43, \mathrm{CHCl}_{3}\right) .{ }^{1} \mathrm{H}-\mathrm{NMR}$ : $2.94(1 \mathrm{H}, \mathrm{dd}, J=3.7,9.4 \mathrm{~Hz}, \mathrm{OH}), 3.87\left(1 \mathrm{H}, \mathrm{d}, J=11.9 \mathrm{~Hz}, \mathrm{CH}_{2}\right), 3.89(1 \mathrm{H}$, d, $\left.J=12.4 \mathrm{~Hz}, \mathrm{CH}_{2}\right), 3.92\left(1 \mathrm{H}, \mathrm{d}, J=11.9 \mathrm{~Hz}, \mathrm{CH}_{2}\right), 3.96(1 \mathrm{H}, \mathrm{d}, J=12.4 \mathrm{~Hz}$, $\left.\mathrm{CH}_{2}\right), 4.07\left(1 \mathrm{H}, \mathrm{d}, J=10.7 \mathrm{~Hz}, \mathrm{CH}_{2}\right), 4.16\left(1 \mathrm{H}, \mathrm{dd}, J=3.7,12.2 \mathrm{~Hz}, \mathrm{CH}_{2}\right)$, $4.20\left(1 \mathrm{H}, \mathrm{d}, J=10.7 \mathrm{~Hz}, \mathrm{CH}_{2}\right), 4.28\left(1 \mathrm{H}, \mathrm{dd}, J=9.4,12.2 \mathrm{~Hz}, \mathrm{CH}_{2}\right), 5.06$ $\left(1 \mathrm{H}, \mathrm{s}, \mathrm{CH}_{2}\right), 5.19\left(1 \mathrm{H}, \mathrm{s}, \mathrm{CH}_{2}\right), 7.04(1 \mathrm{H}, \mathrm{d}, J=8.5 \mathrm{~Hz}, \mathrm{ArH}), 7.07(1 \mathrm{H}, \mathrm{d}$, $J=8.5 \mathrm{~Hz}, \mathrm{ArH}), 7.24(2 \mathrm{H}, \mathrm{m}, \mathrm{ArH}), 7.47(2 \mathrm{H}, \mathrm{m}, \mathrm{ArH}), 7.69(1 \mathrm{H}, \mathrm{d}, J=8.5$ $\mathrm{Hz}, \mathrm{ArH}), 7.79$ (1H, d, $J=8.5 \mathrm{~Hz}, \mathrm{ArH}), 7.94(1 \mathrm{H}, \mathrm{d}, J=8.5 \mathrm{~Hz}, \mathrm{ArH}), 7.94$ $(1 \mathrm{H}, \mathrm{d}, J=8.5 \mathrm{~Hz}, \mathrm{ArH}), 8.02(2 \mathrm{H}, \mathrm{m}, \mathrm{ArH}) .{ }^{13} \mathrm{C}-\mathrm{NMR}: 44.9,63.2,71.0$, $71.1,117.6,126.0,126.1,126.29,126.34,126.5,126.7,127.4,127.6$, $128.06,128.08,128.5,128.7,132.7,133.0,133.1,133.3,133.5,133.8$, 135.4, 138.1, 141.2. IR (Nujol): $3400,1595 \mathrm{~cm}^{-1}$. MS $m / z$ : $402\left(\mathrm{M}^{+}\right)$. HRMS $m / z$ : Calcd for $\mathrm{C}_{26} \mathrm{H}_{23} \mathrm{ClO}_{2:}$ 402.1387. Found: 402.1381 .

(R)-10-Methylene7,10,11,13-tetrahydro-9H-dinaphtho[2,1- $g$ :1,2i][1,5]dioxacycloundecine (21) A solution of $20(470 \mathrm{mg}, 1.20 \mathrm{mmol})$ in DMF $(45 \mathrm{ml})$ was added to a suspension of sodium hydride $(60 \%, 56 \mathrm{mg}$, $1.40 \mathrm{mmol})$ in DMF $(140 \mathrm{ml})$ at $0{ }^{\circ} \mathrm{C}$ over a period of $5 \mathrm{~h}$. The whole was stirred at room temperature for $24 \mathrm{~h}$ and was quenched with satd $\mathrm{NH}_{4} \mathrm{Cl}$. The mixture was extracted with benzene $(30 \mathrm{ml})$. The combined organic layers were washed with brine. Concentration and column chromatography (acetone/hexane $=1 / 20)$ gave $21(69 \mathrm{mg}, 15 \%)$ as a white amorphous of $[\alpha]_{\mathrm{D}}^{25}$ $+278^{\circ}\left(c=0.18, \mathrm{CHCl}_{3}\right) .{ }^{1} \mathrm{H}-\mathrm{NMR}: 3.76\left(2 \mathrm{H}, \mathrm{d}, J=11.9 \mathrm{~Hz}, \mathrm{CH}_{2}\right), 4.08(2 \mathrm{H}$, d, $\left.J=11.9 \mathrm{~Hz}, \mathrm{CH}_{2}\right), 4.23\left(2 \mathrm{H}, J=11.8 \mathrm{~Hz}, \mathrm{CH}_{2}\right), 4.46(2 \mathrm{H}, J=11.8 \mathrm{~Hz}$, $\left.\mathrm{CH}_{2}\right), 4.87\left(2 \mathrm{H}, \mathrm{s}, \mathrm{CH}_{2}\right), 7.04(2 \mathrm{H}, \mathrm{d}, J=8.0 \mathrm{~Hz}, \mathrm{ArH}), 7.22(2 \mathrm{H}, \mathrm{dd}, J=7.3$, $7.3 \mathrm{~Hz}, \mathrm{ArH}), 7.44(2 \mathrm{H}, \mathrm{dd}, J=7.3,7.3 \mathrm{~Hz}, \mathrm{ArH}), 7.68(2 \mathrm{H}, \mathrm{d}, J=8.4 \mathrm{~Hz}$, $\mathrm{ArH}), 7.92(2 \mathrm{H}, \mathrm{d}, J=8.0 \mathrm{~Hz}, \mathrm{ArH}), 7.98(2 \mathrm{H}, \mathrm{d}, J=8.4 \mathrm{~Hz}, \mathrm{ArH}) .{ }^{13} \mathrm{C}-\mathrm{NMR}$ : 70.6, 71.2, 113.6, 125.8, 125.9, 126.0, 126.4, 128.0, 128.1, 128.2, 132.8, $133.3,135.2,145.0$. IR (Nujol): $1640 \mathrm{~cm}^{-1}$. MS $\mathrm{m} / z: 366\left(\mathrm{M}^{+}\right)$. HR-MS $m / z$ : Calcd for $\mathrm{C}_{26} \mathrm{H}_{22} \mathrm{O}_{2}: 366.1620$. Found: 366.1626 .

(R)-7,13-Dihydro-9H-dinaphtho[2,1-g:1,2-i][1,5]dioxacycloundecine10(11 H)-one (8) To a suspension of $21(42 \mathrm{mg}, 0.1 \mathrm{mmol})$ and $\mathrm{K}_{2} \mathrm{OsO}_{4}$. $2 \mathrm{H}_{2} \mathrm{O}(4 \mathrm{mg}, 0.01 \mathrm{mmol})$ in THF $(2.4 \mathrm{ml})$ was added a solution of sodium metaperiodate $(99 \mathrm{mg}, 0.5 \mathrm{mmol})$ in water $(0.8 \mathrm{ml})$. The whole was stirred at room temperature for $1 \mathrm{~d}$. After addition of water $(10 \mathrm{ml})$, the mixture was extracted with TBME $(10 \mathrm{ml})$. The extract was washed with brine. Concen- 
tration and column chromatography (AcOEt/hexane/TEA $=2 / 20 / 1)$ gave 8 $(42 \mathrm{mg}, 99 \%)$ as a solid of $\mathrm{mp} 171.0-172.5^{\circ} \mathrm{C}$ and $[\alpha]_{\mathrm{D}}^{25}+257^{\circ}(c=0.32$, $\left.\mathrm{CHCl}_{3}\right) .{ }^{1} \mathrm{H}-\mathrm{NMR}: 3.86\left(2 \mathrm{H}, \mathrm{d}, J=14.1 \mathrm{~Hz}, \mathrm{CH}_{2}\right), 4.07(2 \mathrm{H}, \mathrm{d}, J=14.1 \mathrm{~Hz}$, $\left.\mathrm{CH}_{2}\right), 4.30\left(2 \mathrm{H}, \mathrm{d}, J=10.5 \mathrm{~Hz}, \mathrm{CH}_{2}\right), 4.55\left(2 \mathrm{H}, \mathrm{d}, J=10.5 \mathrm{~Hz}, \mathrm{CH}_{2}\right), 7.02$ $(2 \mathrm{H}, \mathrm{d}, J=8.6 \mathrm{~Hz}, \mathrm{ArH}), 7.23(2 \mathrm{H}, \mathrm{ddd}, J=1.1,8.6,8.6 \mathrm{~Hz}, \mathrm{ArH}), 7.47(2 \mathrm{H}$, ddd, $J=1.1,8.3,8.3 \mathrm{~Hz}, \mathrm{ArH}), 7.68(2 \mathrm{H}, \mathrm{d}, J=8.6 \mathrm{~Hz}, \mathrm{ArH}), 7.94(2 \mathrm{H}, \mathrm{d}$, $J=8.3 \mathrm{~Hz}, \mathrm{ArH}), 8.01(2 \mathrm{H}, \mathrm{d}, J=8.6 \mathrm{~Hz}, \mathrm{ArH}) .{ }^{13} \mathrm{C}-\mathrm{NMR}: 73.0,73.4,126.2$, $126.3,126.6,128.0,128.2,128.4,132.9,133.4,134.0,135.4,208.6$. IR (Nujol): $1725 \mathrm{~cm}^{-1}$. MS m/z: $368\left(\mathrm{M}^{+}\right)$. HR-MS $m / z$ : Calcd for $\mathrm{C}_{25} \mathrm{H}_{20} \mathrm{O}_{3}$ : 368.1412. Found: 368.1402 .

Asymmetric Epoxidation of Stilbene Catalyzed by 4 (Table 1, Entry 7) To an acetonitrile solution $(7.5 \mathrm{ml})$ of trans-stilbene $(90 \mathrm{mg}, 0.50 \mathrm{mmol}), 4$ (65 mg, $0.15 \mathrm{mmol})$, and an aqueous $\mathrm{Na}_{2}$ EDTA solution $\left(3 \mathrm{ml}, 4 \times 10^{-4} \mathrm{M}\right)$ was added a mixture of oxone ${ }^{\circledR}(922 \mathrm{mg}, 1.5 \mathrm{mmol})$ and sodium bicarbonate $(391 \mathrm{mg}, 4.7 \mathrm{mmol})$ in portions over a period of $1 \mathrm{~h}$ at room temperature (r.t.). After stirring for $1 \mathrm{~h}$, the mixture was poured onto water $(20 \mathrm{ml})$, and extracted with $\mathrm{CHCl}_{3}(60 \mathrm{ml})$. The combined organic layers were washed with brine. Concentration and column chromatography (hexane, then hexane/AcOEt=9/1) gave trans-stilbene epoxide $(74 \mathrm{mg}, 78 \%)$ of $[\alpha]_{\mathrm{D}}^{25}$ $-47.6^{\circ}\left(c=1.19, \mathrm{CHCl}_{3}\right)$ in $18 \%$ ee. ${ }^{1} \mathrm{H}-\mathrm{NMR}, \mathrm{IR}$, and MS were identical with those reported. ${ }^{14)}$ The ee was determined by HPLC analysis using a chiral stationary phase column (Daicel Chiralcel OD-H, hexane/iso-PrOH= $9: 1,0.5 \mathrm{ml} / \mathrm{min}, 15.0 \mathrm{~min}$ (major enantiomer), $26.9 \mathrm{~min}$ (miner enantiomer). In entry 12 , column chromatography gave $(R)$-7,14-dihydrodinaphtho[2,1$h: 1,2-j][1,3,6]$ trioxacyclododecin-11(12H)-one 22 as a white gum in $55 \%$ yield: ${ }^{1} \mathrm{H}-\mathrm{NMR}: 3.85\left(1 \mathrm{H}, \mathrm{d}, J=12.5 \mathrm{~Hz}, \mathrm{CH}_{2}\right), 3.89(1 \mathrm{H}, \mathrm{d}, J=16.2 \mathrm{~Hz}$, $\left.\mathrm{CH}_{2}\right), 4.23\left(1 \mathrm{H}, \mathrm{d}, J=16.2 \mathrm{~Hz}, \mathrm{CH}_{2}\right), 4.30\left(1 \mathrm{H}, J=12.2 \mathrm{~Hz}, \mathrm{CH}_{2}\right), 4.67(1 \mathrm{H}$, $\left.J=12.5 \mathrm{~Hz}, \mathrm{CH}_{2}\right), 4.77\left(1 \mathrm{H}, J=12.2 \mathrm{~Hz}, \mathrm{CH}_{2}\right), 4.78\left(1 \mathrm{H}, J=5.8 \mathrm{~Hz}, \mathrm{CH}_{2}\right)$, $5.73\left(1 \mathrm{H}, J=5.8 \mathrm{~Hz}, \mathrm{CH}_{2}\right), 6.95(1 \mathrm{H}, \mathrm{d}, J=8.6 \mathrm{~Hz}, \mathrm{ArH}), 6.95(1 \mathrm{H}, \mathrm{d}$, $J=8.6 \mathrm{~Hz}, \mathrm{ArH}), 7.23(2 \mathrm{H}, \mathrm{m}, \mathrm{ArH}), 7.45(1 \mathrm{H}, \mathrm{d}, J=7.3 \mathrm{~Hz}, \mathrm{ArH}), 7.47(1 \mathrm{H}$, d, $J=7.3 \mathrm{~Hz}, \mathrm{ArH}), 7.75(1 \mathrm{H}, \mathrm{d}, J=8.6 \mathrm{~Hz}, \mathrm{ArH}), 7.87(1 \mathrm{H}, \mathrm{d}, J=8.6 \mathrm{~Hz}$, ArH), 7.92 (1H, d, $J=8.6 \mathrm{~Hz}, \mathrm{ArH}), 7.94(1 \mathrm{H}, \mathrm{d}, J=8.3 \mathrm{~Hz}, \mathrm{ArH}), 8.01$ (1H, d, $J=8.5 \mathrm{~Hz}, \mathrm{ArH}), 8.06(1 \mathrm{H}, \mathrm{d}, J=8.6 \mathrm{~Hz}, \mathrm{ArH}) .{ }^{13} \mathrm{C}-\mathrm{NMR}: 69.2,69.4$, $71.3,88.7,126.4,126.8,127.0,127.1,127.4,127.5,127.76,127.83,129.3$, $129.4,129.6,129.7,133.3,134.3,134.39,134.42,134.47,134.53,134.6$, 135.9, 172.0. IR $\left(\mathrm{CHCl}_{3}\right): 1740 \mathrm{~cm}^{-1}$. MS $m / z: 384\left(\mathrm{M}^{+}\right)$. HR-MS $m / z$ : Calcd for $\mathrm{C}_{25} \mathrm{H}_{20} \mathrm{O}_{4}$ : 384.1362 . Found: 384.1368 .

Acknowledgments This research was supported by a Grant-in-Aid for Scientific Research on Priority Areas (A) "Exploitation of Multi-Element Cyclic Molecules" from the Ministry of Education, Culture, Sports, Science and Technology, Japan.

\section{References and Notes}

1) a) Fujihara H., Nagai K., Tomioka K., J. Am. Chem. Soc., 122, 12055-12056 (2000); b) Nishimura K., Ono M., Nagaoka Y., Tomioka K., Angew. Chem. Int. Ed., 40, 440-442 (2001).

2) For reviews on chiral ligand-promoted asymmetric reactions, see: $a$ ) Tomioka K., Synthesis, 1990, 541—549; b) Noyori R., "Asymmetric Catalysis in Organic Synthesis," John Wiley and Sons, Inc., New York, 1994; c) Seyden-Penne J., "Chiral Auxiliaries and Ligands in Asymmetric Synthesis,” John Wiley and Sons, Inc., New York 1995.

3) Leading references on an asymmetric epoxidation with chiral ketones. Curci R., Fiorentino M., Serio M. R., J. Chem. Soc., Chem. Commun., 1984, 155-156; Curci R., D’Accolti L., Fiorentino M., Rosa A., Tetrahedron Lett., 36, 5831-5834 (1995); Denmark S. E., Forbes D. C., Hays D. S., DePue J. S., Wilde R. G., J. Org. Chem., 60, 13911407 (1995); Brown D. S., Marples B. A., Smith P., Walton L., Tetrahedron, 51, 3587-3606 (1995); Yang D., Yip Y. C., Tang M. W., Wong M. K., Zheng J. H., Cheung K. K., J. Am. Chem. Soc., 118, 491-492 (1996); Tu Y., Wang Z.-X., Shi Y., ibid., 118, 9806-9807 (1996); Yang D., Wang X.-C., Wong M.-K., Yip Y.-C., Tang M.-W., ibid., 118, $11311-11312$ (1996); h) Wang Z.-X., Tu Y., Frohn M., Shi Y., J. Org. Chem., 62, 2328-2329 (1997); Denmark S. E., Wu Z.,
Crudden C. M., Matsuhashi H., ibid., 62, 8288-8289 (1997); Wang Z. X., Tu Y., Frohn M., Zhang J. R., Shi Y., J. Am. Chem. Soc., 119, 11224-11235 (1997); Wang Z.-X., Shi Y., J. Org. Chem., 62, 86228623 (1997); Zhao C. G., Adam W., Tetrahedron: Asymmetry, 8, 3995-3998 (1997); Adam W., Fell R. T., Saha-Moller C. R., Zhao C.G., ibid., 9, 397-401 (1998); Song C. E., Kim Y. H., Lee K. C., Lee S. G., Jin B. W., ibid., 8, 2921-2926 (1997); Armstrong A., Hayter B. R., Chem. Commun., 1998, 621-622; Frohn M., Dalkiewicz M., Tu Y., Wang Z.-X., Shi Y., J. Org. Chem., 63, 2948-2953 (1998); Wang Z.-X., Shi Y., ibid., 63, 3099-3104 (1998); Cao G.-A., Wang Z.-X., Tu Y., Shi Y., Tetrahedron Lett., 39, 4425-4428 (1998); Yang D., Wong M.-K., Yip Y.-C., Wang X.-C., Tang M.-W., Zheng J.-H., Cheung K.-K., J. Am. Chem. Soc., 120, 5943-5952 (1998); Zhu Y., Tu Y., Yu H., Shi Y., Tetrahedron Lett., 39, 7819-7822 (1998); Tu Y., Wang Z.-X., Frohn M., He M., Yu H., Tang Y., Shi Y., J. Org. Chem., 63, 8475-8485 (1998); Yang D., Yip Y.-C., Chen J., Cheung K.-K., J. Am. Chem. Soc., 120, 7659-7660 (1998); Adam W., Saha-Moller C. R., Zhao C.-G., Tetrahedron: Asymmetry, 10, 2749-2755 (1999); Wang Z.-X., Miller S. M., Anderson O. P., Shi Y., J. Org. Chem., 64, 6443-6458 (1999); Carnell A. J., Johnstone R. A. W., Parsy C. C., Sanderson W. R., Tetrahedron Lett., 40, 8029-8032 (1999); Armstrong A., Hayter B. R., Tetrahedron, 55, 11119-11126 (1999); Wang Z.-X., Cao G.-A., Shi Y., J. Org. Chem., 64, 7646-7650 (1999); Warren J. D., Shi Y., ibid., 64, 7675-7677 (1999); Frohn M., Zhou X., Zhang J.-R., Tang Y., Shi Y., J. Am. Chem. Soc., 121, 7718 -7719 (1999); Shu L., Shi Y., Tetrahedron Lett., 40, 87218724 (1999); Armstrong A., Hayter B. R., Moss W. O., Reeves J. R., Wailes J. S., Tetrahedron: Asymmetry, 11, 2057-2061 (2000); Solladie-Cavallo A., Bouerat L., Organic Lett., 2, 3531-3534 (2000); Tian H., She X., Xu J., Shi Y., ibid., 3, 1929-1931 (2001).

4) For a recent review on asymmetric epoxidation of electron-deficient olefins, see: Porter M. J., Skidmore J., Chem. Commun., 2000, 12151225 .

5) a) Shindo M., Koga K., Tomioka K., J. Org. Chem., 63, 9351-9357 (1998); b) Mori T., Kosaka K., Nakagawa Y., Nagaoka Y., Tomioka K., Tetrahedron: Asymmetry, 9, 3175-3178 (1998); c) Tomioka K., Fujieda H., Hayashi S., Hussein M. A., Kambara T., Nomura Y., Kanai M., Koga K., Chem. Commun., 715-716 (1999); d) Hussein M. A., Iida A., Tomioka K., Tetrahedron, 55, 11219-11228 (1999).

6) a) Murray R. W., Chem. Rev., 89, 1187-1201 (1989); b) Adam W., Curci R., Edwards J. O., Acc. Chem. Res., 22, 205-211 (1989); c) Curci R., Dinoi A., Rubino M. F., Pure Appl. Chem., 67, 811-822 (1995).

7) Matsumoto K., Tomioka K, Heterocycles, 54, 615-617 (2001).

8) Quinkert G., Grosso M. D., Doring A., Doring W., Schenkel R. I., Bauch M., Dambacher G. T., Bats J. W., Zimmermann G., Durner G., Helv. Chim. Acta, 78, 1345-1389 (1995).

9) Whitney T. A., J. Org. Chem., 45, 4214-4216 (1980)

10) Ohta T., Ito M., Inagaki K., Takaya H., Tetrahedron Lett., 34, 16151616 (1993).

11) Rosini C., Tanturli R., Pertici P., Salvadori P., Tetrahedron: Asymmetry, 7, 2971-2982 (1996).

12) The reaction was monitored by TLC. The extracted organic layers were dried over $\mathrm{Na}_{2} \mathrm{SO}_{4}$ unless otherwise noted. Purification was carried out using silica gel column chromatography unless otherwise noted. ${ }^{1} \mathrm{H}-\mathrm{NMR}(500 \mathrm{MHz})$ and ${ }^{13} \mathrm{C}-\mathrm{NMR}(126 \mathrm{MHz})$ were measured in $\mathrm{CDCl}_{3}$ unless otherwise noted. Chemical shift $(\delta)$ was presented in ppm relative to internal tetramethylsilane. Coupling constant value $(J)$ was presented in Hz. Mass spectra were measured by electron impact (EI) mode unless otherwise noted.

13) Takahashi H., Kawakita T., Ohno M., Yoshioka M., Kobayashi S., Tetrahedron, 48, 5691-5700 (1992).

14) Chang H. T., Sharpless K. B., J. Org. Chem., 61, 6456-6457 (1996). 